\title{
Geometrie der Zahlen
}

\section{Zum 150. Geburtstag von Hermann Minkowski}

\author{
Yuri Tschinkel
}

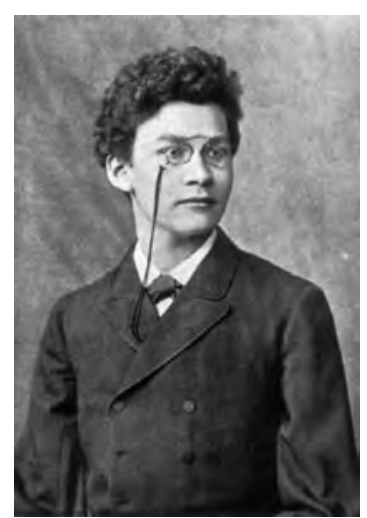

Hermann Minkowski
Heers Hermann Minkowski wurde vor 150 Jahren, am 22. Juni 1864 geboren. Er entstammte einer jüdischen Familie mit Wurzeln in Osteuropa. Einer seiner Vorfahren war Baruch ben Jakob aus Shklov, ein Richter in Minsk, mit Neigung zur Medizin. Nach umfangreichen Reisen durch England, Holland und Deutschland, kehrte dieser nach Weißrussland zurück und starb dort in Sluzk im Jahr 1812. Er übersetzte die ersten sechs Bände von Euklid aus dem Griechischen ins Hebräische, in der Hoffnung, diesen klassischen Text zugänglicher zu machen. Sein Enkel, Isaak ben Aaron, nahm den Namen Minkowski an und lebte in einer kleinen Stadt Karlin (Pinsk), die zuerst Polnisch, dann Schwedisch, dann Russisch war, und gegenwärtig in Weißrussland liegt. Isaaks Enkel Levin (18251884), ein Weizenhändler in Vilna, heiratete Rahel, die Tochter eines Weizenhändlers. Sie lebten in Alexotas (PolnischLittauisch, Preußisch, Polnisch, und Russisch) und hatten fünf Kinder: Max, Oscar, Fanny, Hermann und Toby. Um dem wachsenden Antisemitismus im zaristischen Russland zu entkommen, übersiedelte die Familie nach Königsberg und wurde 1872 in Preußen naturalisiert.

Hermann Minkowski wurde dort auf das Altstädtische Gymnasium aufgenommen, an dem er mit 15 Jahren sein Abitur ablegte. Dieses Gymnasium existierte schon im 16. Jahrhundert und war eine Art Ostpreußische "Spezialschule“: Es publizierte eigene Berichte und hatte u. a. das Crellsche Journal für die Reine und Angewandte Mathematik in der Schulbibliothek (was heute sogar für Mathematikinstitute keine Selbstverständlichkeit ist).

Nach Studien an den Universitäten Königsberg und Berlin, der Promotion in Königsberg 1885, dem Armeedienst sowie Anstellungen an den Universitäten Bonn und Königsberg, wurde Minkowski 1896 auf eine Ordentliche Professur am Eidgenössischen Polytechnikum Zürich (ETH) berufen. Von 1902 bis 1909 war er Professor in Göttingen, wo er am 12. Januar 1909, im Alter von 44 Jahren, an einer Blinddarmentzündung verstarb.

Eine kurze Mitteilung darüber erschien im Jahresbericht der DMV:

Mathemativohe Gesellschaft in Göttingen. Newnte Sitzung am 12. Jamuar 1909: Nach Mitteilnng des am Mittag plötzlich erfolgten Todes von Prof. Mínkowski wird die Sitzung aufgehoben. - Zehnte Sitnung am
Es folgen Zeilen aus dem ergreifenden Nachruf David Hilberts in den Nachrichten der Gesellschaft der Wissenschaften zu Göttingen, 1909:

Mehr als sechs Jahre hindurch haben wir, seine nächsten mathematischen Kollegen, jeden Donnerstag pünktlich drei Uhr mit ihm zusammen einen mathematischen Spaziergang auf den Hainberg gemacht - auch den letzten Donnerstag vor seinem Tode, wo er uns mit besonderer Lebhaftigkeit von den neuen Fortschritten seiner elektrodynamischen Untersuchungen erzählte: den Donnerstag darauf wiederum um drei Uhr - gaben wir ihm das letzte Geleit.

Die Grabstätte von Minkowski und seinem Bruder Oskar (der Arzt und Wissenschaftler entdeckte den Zusammenhang zwischen Bauchspeicheldrüse und Diabetes) befindet sich auf dem Waldfriedhof Heerstraße in BerlinCharlottenburg. Bis 2014 war es eine Ehrengrabstätte der Stadt Berlin. Trotz intensiver Bemühungen der Berliner Mathematiker hat das Land Berlin im Juli 2014 die Anerkennung der Grabstätte der Brüder Minkowski nicht verlängert:

Unter diesen Prämissen war die für Sie - und andere - überraschende Entscheidung einer Nichtverlängerung, wie in anderen vergleichbaren Fällen, folgerichtig, denn bezüglich der hochverdienten Brüder Minkowski war eine solche Popularität, wie sie die Anerkennungskriterien unabdingbar verlangen, leider nicht feststellbar.

\section{Quadratische Formen}

Im Jahr 1882 formulierte die Pariser Académie als Preisaufgabe, das von Ferdinand Gotthold Max Eisenstein 1847 im Crellschen Journal gestellte Problem zu lösen:

17.

Note sur la représentation d'un nombre par la somme de cinq carrés. (Par Mr. G, Eisenstein.)

Mr. Dirichlet, à la fin de son beau mémoire sur les formes quadratiques *), a remsrqué que par la combinaison de deax théories, dont on doit lune ù luimème, l'autre à Mr. Gau/s, on peut trouver des expressions très-simples et trèsremarquables du nombre des représentations d'un entier donné par la somme de trois carrés. Jai trouvé qu'il existe des formules nbsolument semblables pour le

Es ging um die Anzahl der Darstellungen einer natürlichen Zahl als Summe von fünf Quadratzahlen, also um ganzzahlige Lösungen der Gleichung

$$
m=x_{1}^{2}+x_{2}^{2}+x_{3}^{2}+x_{4}^{2}+x_{5}^{2}, \quad m \in \mathbb{N} .
$$


Minkowski erhielt die Auszeichnung der Académie und wurde über Nacht international berühmt. Es gab allerdings gewisse sonderbare Umstände, die die Entscheidung der Académie begleiteten: Die Académie übersah, dass ein führender englischer Mathematiker, Henry John Steven Smith, das Problem schon 1868 in den Proceedings of the Royal Society of London behandelt hatte. Smith war nicht wenig erstaunt, dass seine Arbeit bei den französischen Mathematikern ohne Beachtung blieb, und beschwerte sich bei Hermite. Hermite versicherte, dass die Verlegenheit ganz bei der Académie-Kommission liege und dass es nur eines Manuskripts von Smith bedürfte, um die Verlegenheit aus der Welt zu räumen. Am Ende wurde das Preisgeld zwischen Minkowski und dem bis dahin verstorbenen Smith geteilt.

Der Wettbewerb mit französischer Mathematik blieb allgegenwärtig:

Minkowski ist es zu danken, daß nach Hermite's Tode die Führerrolle in der Zahlentheorie wieder in deutsche Hände zurückfiel, und wenn man überhaupt bei einer solchen Wissenschaft, wie es die Arithmetik ist, die Beteiligung der Nationen an den Fortschritten und Errungenschaften abwägen will: wesentlich durch Minkowskis Wirken ist es gekommen, daß heute im Reiche der Zahlen die bedingungslose und unbestrittene deutsche Vorherrschaft statthat. (Hilbert: Nachruf auf Minkowski)

Bonn

Trotz des frühen Erfolgs ergab sich die Beförderung zum Ordentlichen Professor nicht automatisch. Der Briefwechsel mit Hilbert verrät Nervosität. Insbesondere war Minkowski von Bonn nicht sehr begeistert [9, S. 54]:

Der Umgang hier mit meinen mathematischen Kollegen ist wirklich bejammernswert; der eine klagt über Migräne, so wie man $a$ oder $x$ sagt; bei dem anderen tritt innerhalb von fünf Minuten die Frau dazwischen, um dem Gespräch eine andere Wendung zu geben.

Auch die Rückkehr nach Königsberg und die Ernennung zum Ordentlichen Professor im Jahr 1895 lässt ihn rastlos. So wird ein Ruf nach Zürich zu einer großen Freude:

In Zürich selbst ist es, wie Du Dir denken kannst, wunderschön. [9, S. 85]

Die komplizierten Verhandlungen sind erfolgreich, und seine neuen Vorgesetzten voll des Lobes:

Die Vielseitigkeit seines Wissens geht auch aus der Liste der bis jetzt von ihm in Bonn und Königsberg gehaltenen Vorlesungen hervor, die fast den ganzen Umfang der heutigen mathematischen Forschung und ihrer Anwendungen auf Mechanik und Physik berühren. Ich will nicht versäumen hervorzuheben, daß der Eindruck, den der erst 32-jährige Mann auch außerhalb seiner Wissenschaft macht, ein ganz bedeutender und sehr sympatischer ist. (Brief an den Bundesrat, September 1896, siehe [12].)
Die frühe Begeisterung für Zürich leidet an den Realitäten des Alltags. Minkowski klagt über das schwache Niveau der Hörer (unter seinen Studenten war auch Einstein):

Schliesslich komme ich noch gar in den Ruf eines schwierigen Docenten, und dann sind von vornherein die Meldungen zu den Vorlesungen wenig zahlreich. Ich werde in der Popularisierung des Stoffs bis an die äusserst mögliche Grenze gehen müssen; denn diejenigen, die mich vielleicht um wissenschaftlicher Leistungen wegen genommen haben, wollen schliesslich für ihr Geld auch etwas haben. So sehe ich ziemlich traurig in die weiteren Semester, viel solche Arbeit, die mir im Grunde wenig zu Gute kommt, anders, als ich mir die Sachlage bei Annahme des Rufes ausgemalt habe. [9, S. 94]

Aber auch mit Kollegen scheint es Kontaktschwierigkeiten zu geben:

Auch die eigentlichen Mathematiker, deren Zahl aber sehr gering ist, sind durch alle Collegien, die sie sonst hören müssen, so in Anspruch genommen, dass sie nur geniesen können, was ihnen zerschnitten und zerlegt nach gewaltsamer Öffnung des Mundes eingetrichtert wird.

Die Kollegen delegieren Minkowski in das Vergnügungskomitee des ersten Internationalen Mathematikerkongresses in Zürich.

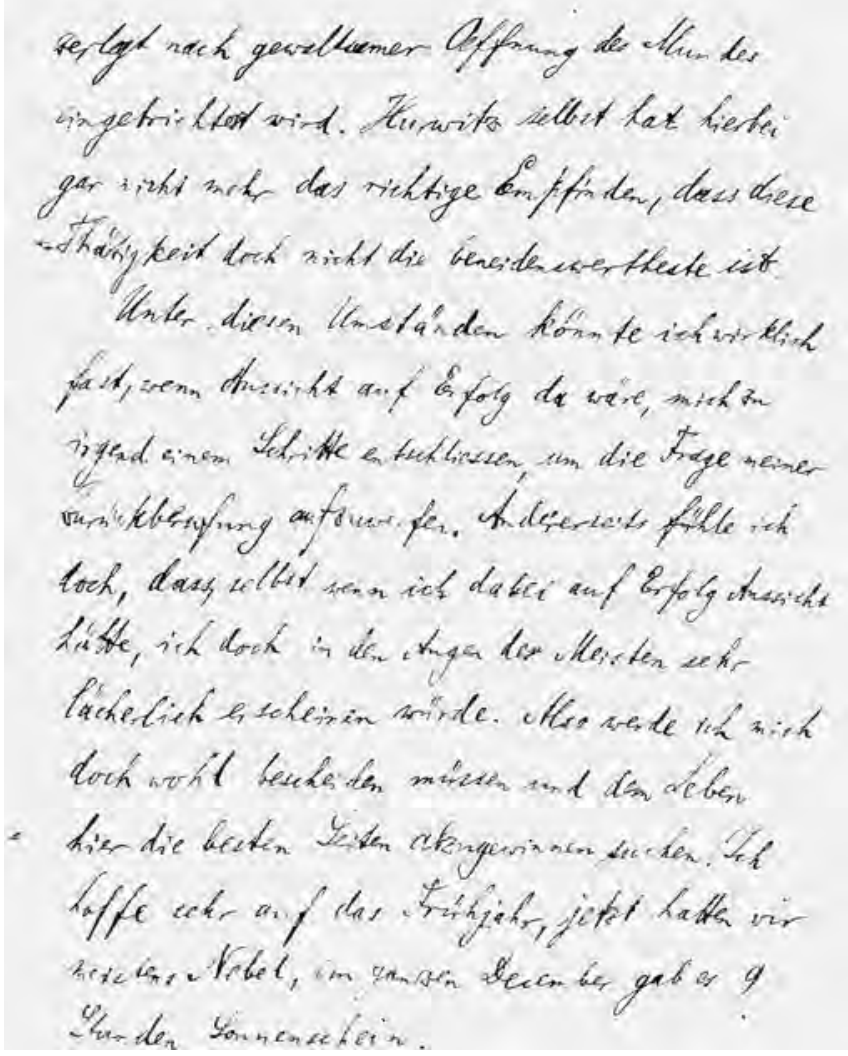

„... was ihnen zerschnitten und zerlegt nach gewaltsamer Öffnung des Mundes eingetrichtert wird " 


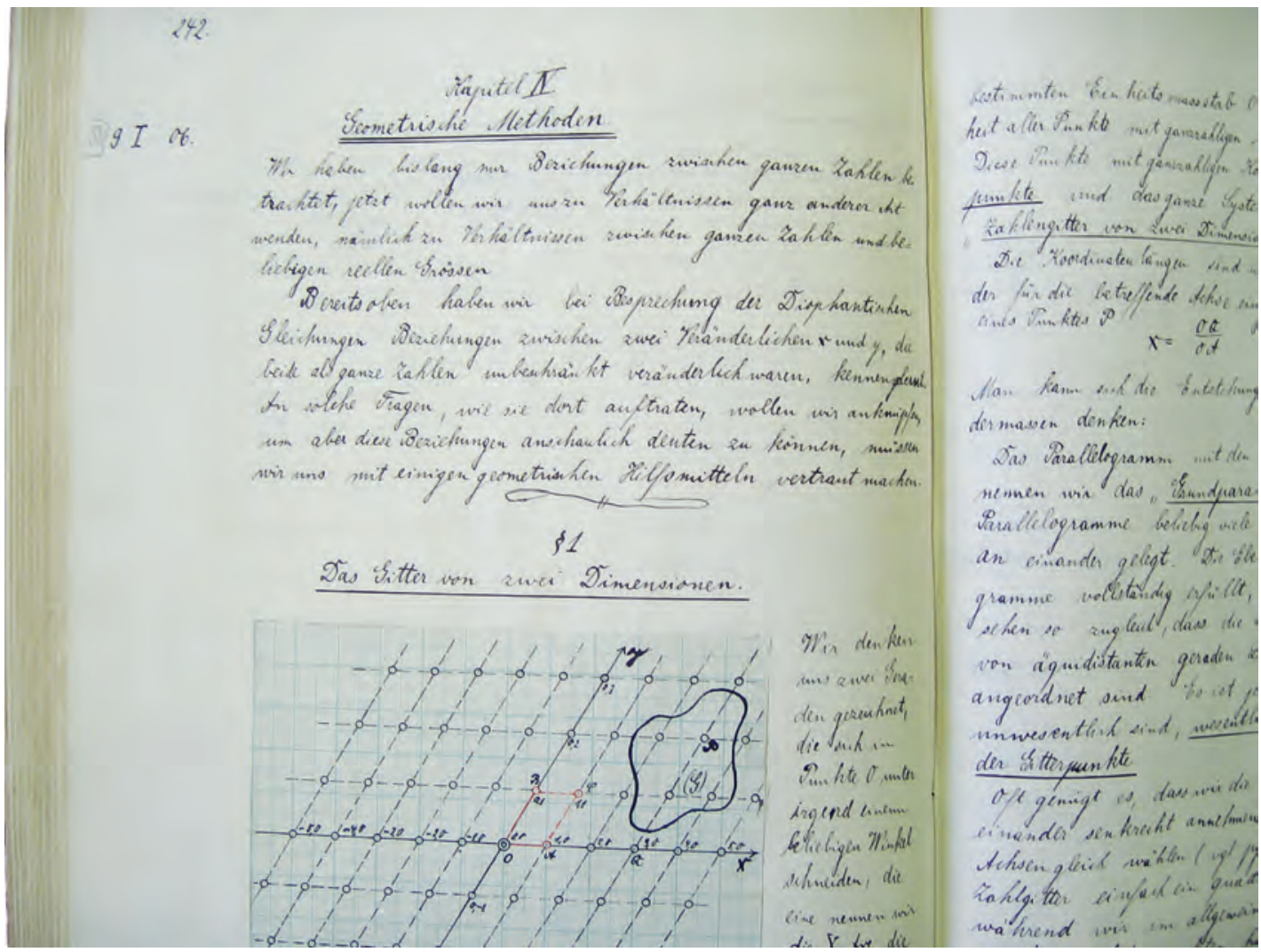

Vorlesungsmitschrift, Göttingen (Niedersächsische Staats- und Universitätsbibliothek Göttingen)

\section{Moderne Zeiten}

\section{Geometrie der Zahlen}

Die von Minkowski begründete „Geometrie der Zahlen“ ist auch heute ein sehr aktives Forschungsgebiet und kann als Vorreiter der "Arakelov-Geometrie“ betrachtet werden.

Es geht dabei um Wechselwirkungen zwischen starren Objekten, wie Gittern oder ganzzahligen Lösungen diophantischer Gleichungen, und „weicher" Geometrie; um Beziehungen zwischen Fläche, Volumen, Krümmung und Gitterpunkten.

Ein Hauptsatz der Geometrie der Zahlen lautet wie folgt: Sei $\Lambda \subset \mathbb{R}^{2}$ ein Gitter mit Fundamentalgebiet der Fläche 1. Sei $\mathcal{D} \subset \mathbb{R}^{2}$ ein konvexes, zentral-symmetrisches Gebiet der Fläche $>$ 4. Dann enthält $\mathcal{D}$ mindestens zwei Gitterpunkte aus $\Lambda$.

Dieser Satz hat viele Anwendungen und Verallgemeinerungen, zum Beispiel in algebraischer Zahlentheorie. Dort untersucht man Ringe ganzer Zahlen in endlichen Erweiterungen $K$ von $\mathbb{Q}$. Eine wichtige Invariante ist die Diskriminante des Ringes; wenn der Ring $\mathcal{O}_{K} \subset K$ als $\mathbb{Z}[\alpha]$ gegeben werden kann, wo $\alpha$ eine ganze algebraische Zahl ist, ist die Diskriminante

$$
\mathfrak{D}(K):=\prod_{i<j}\left(\alpha_{i}-\alpha_{j}\right)^{2},
$$

wobei $\left\{\alpha_{i}\right\}$ die Menge der Wurzeln des Minimalpolynoms von $\alpha$ ist.
Es wird vermutet, dass die Anzahl der Körper mit beschränkter Diskriminante linear wächst:

$$
\#\{[K: \mathbb{Q}]=d|| \mathfrak{i}(K) \mid \leq B\} \sim C_{d} \cdot B, \quad B \rightarrow \infty .
$$

Diese Behauptung ist trivial für $d=2$, ein klassisches Theorem für $d=3$ (Davenport-Heilbronn, 1971) und eine brilliante Anwendung der Geometrie der Zahlen für $d=4,5$ (Bhargava, 2001). Für $d>5$ hat man z.Z. nur eine Abschätzung:

$$
\ll B^{\exp (C \sqrt{\log d})} \quad \text { (Ellenberg-Venkatesh, 2006). }
$$

\section{Polyeder}

Minkowski interessierte sich auch für „starre Gebilde“ Polyeder.

Hier sind einige moderne Probleme zu diesem Thema. Gegeben sei ein Gitter $\Lambda \subset \mathbb{R}^{d}$ und ein Polyeder $\Pi$ mit Ecken in $\Lambda$. Gesucht ist die Anzahl $\psi(n)$ der Gitterpunkte in $n \cdot \Pi \cap \Lambda$, für $n \rightarrow \infty$. Die Lösung appelliert an die Theorie der torischen Varietäten; diese werden durch monomiale Gleichungen beschrieben. Aus $(\Pi, \Lambda)$ konstruiert man eine torische Varietät $X_{\Pi, \Lambda}$ und ein Geradenbündel L, sodass

$$
\psi(n)=\operatorname{dim} H^{0}\left(X_{\Pi, \Lambda}, n L\right),
$$

die Dimension des Raumes der globalen Schnitte von $n L$ ist. Dann gibt der Satz von Riemann-Roch eine Formel für $\psi(n)$, die ausschließlich von topologischen Invarianten von $X_{\Pi, \Lambda}$ abhängt. 


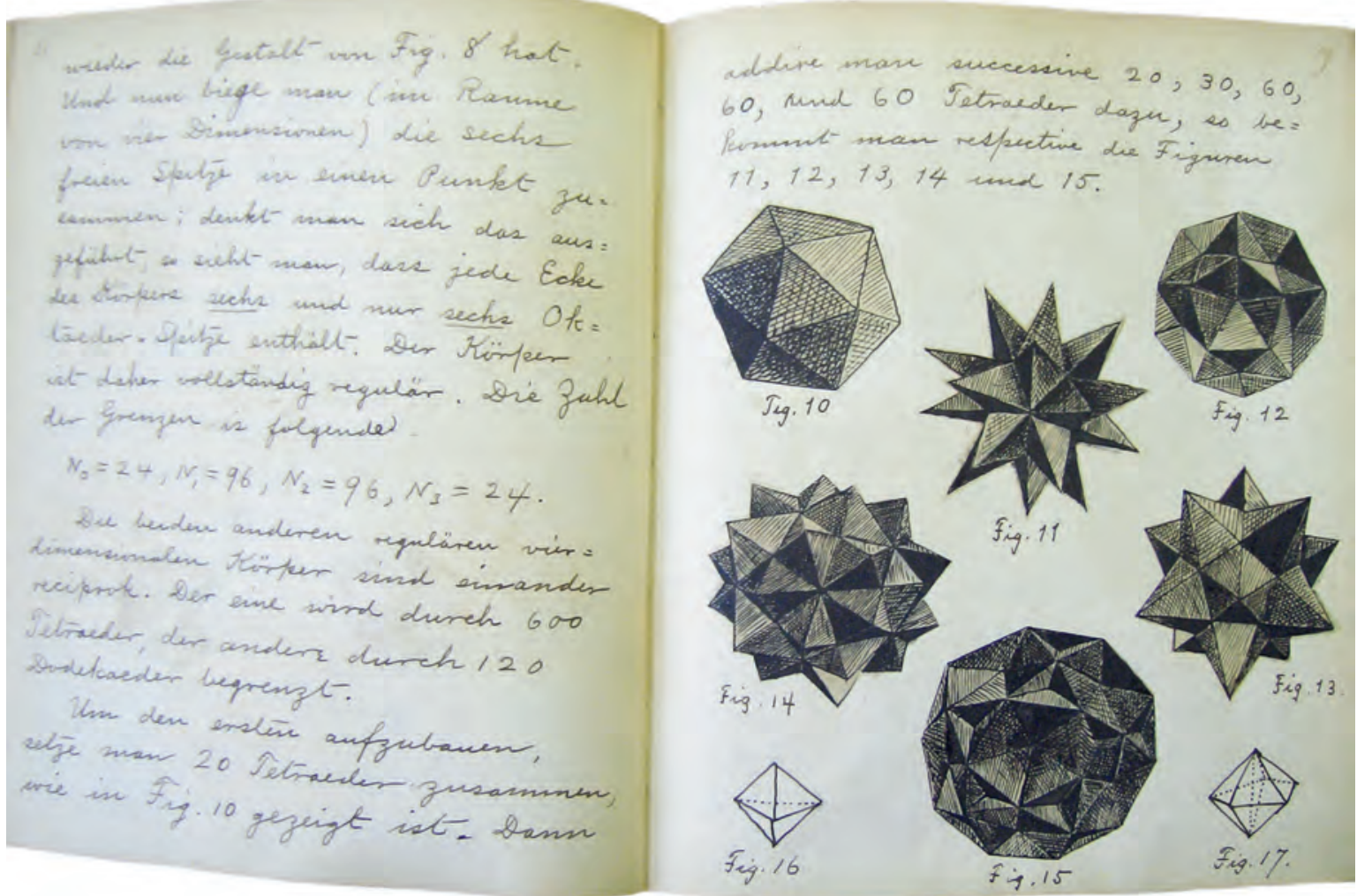

Felix Klein-Protokolle, Göttingen (Niedersächsische Staats- und Universitätsbibliothek Göttingen)

Eine andere kombinatorische Aufgabe in diesem Zusammenhang ist die Untersuchung des $f$-Vektors

$$
f=f(\Pi)=\left(f_{0}(\Pi), f_{1}(\Pi), \ldots, f_{d}(\Pi)\right),
$$

wobei $f_{j}(\Pi)$ die Anzahl der $j$-dimensionalen Seiten von $\Pi$ ist. Auch hier ist der Übergang zu torischen Varietäten von entscheidender Bedeutung: Die Intersection cohomology torischer Varietäten kodiert interessante Beziehungen zwischen $\operatorname{den} f_{j}(\Pi)$.

Weitere Anwendungen von Polyedern finden sich in der Theorie der hypergeometrischen Funktionen, der Darstellungstheorie, in reeller, symplektischer und tropischer Geometrie (Okounkov bodies), in der algebraischen Geometrie bei der Auflösung von Singularitäten, bei der Faktorisierung von birationalen Morphismen, bei Klassifikationsfragen sowie im Programm der Minimalen Modelle (Kegel der amplen und effektiven Divisoren).

\section{Kugelpackungen}

Minkowski beschäftigte sich mit dem Problem der „dichtesten gitterförmigen Lagerung kongruenter Körper", einer Verallgemeinerung der Keplerschen Vermutung.

Die Keplersche Vermutung wurde 1998 von Sam Ferguson und Tom Hales bewiesen. Allerdings stützte sich der Beweis auf umfangreiche Computerberechnungen, die von den Gutachtern der Arbeit nicht im Detail nachvollzogen werden konnten. Erst in diesem Jahr gelang Hales der forma- le Nachweis der Korrektheit des Beweises. (Projekt Flyspeck https://code.google.com/p/flyspeck/)

Über dichteste Packungen mit anderen Körpern, wie zum Beispiel Tetraedern, ist wenig bekannt.

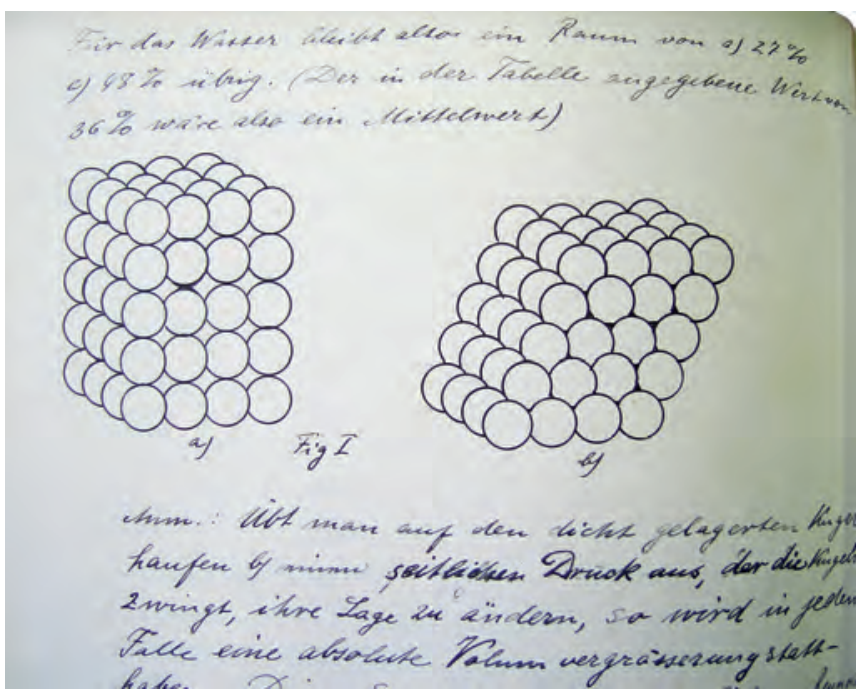

Felix Klein-Protokolle, Göttingen

(Niedersächsische Staats- und Universitätsbibliothek Göttingen) 
Das Hasse-Minkowski-Prinzip

Dieses Prinzip besagt, dass eine quadratische Form $f \in$ $\mathbb{Q}\left[x_{0}, \ldots, x_{n}\right]$, die nicht-triviale Nullstellen in $\mathbb{R}$ und allen $p$-adischen Körpern $\mathbb{Q}_{p}$ hat, auch nicht-triviale Nullstellen in $\mathbb{Q}$ besitzt. Diese Eigenschaft gilt nicht mehr für Formen höheren Grades, z.B. für die Form $3 x_{0}^{3}+4 x_{1}^{3}+5 x_{2}^{3}$.

Eine bemerkenswerte Errungenschaft von M. Bhargava (Fields-Medaille 2014) ist der auf der Geometrie der Zahlen basierende Beweis, dass ein positiver Anteil der kubischen Formen in drei Variablen dem Hasse-Minkowski-Prinzip genügt, und dass ein positiver Anteil der Formen Gegenbeispiele zu diesem Prinzip liefert [3].

Auch in höheren Dimensionen gibt es Gegenbeispiele. So hat die Gleichung

$$
x^{2}+x_{1}^{2}=f(t) x_{2}^{2}, \quad f(t)=\left(t^{2}-2\right)\left(3-t^{2}\right)
$$

keine nicht-trivialen Lösungen in $\mathbb{Q}$. Dies wird mit sogenannten Brauer-Manin-Obstruktionen gezeigt. Ein wirklicher Durchbruch gelang in [6]: Für viele Kegelbündel, wie (1), folgt aus dem Verschwinden der Brauer-ManinObstruktion die Existenz nicht-trivialer rationaler Lösungen. Der Beweis basiert auf Lehrsätzen in additiver Kombinatorik aus der Doktorarbeit von L. Matthiesen, die wiederum aus der Theorie von B. Green und T. Tao folgen.

\section{Flugzeuge}

Um 1905 versuchte Minkowski, eine mathematische Erklärung für das Interferenzexperiment von Michelson zu finden und war sehr überrascht, dass sein Student Einstein schon eine Arbeit darüber publiziert hatte [5], [7]. Die Einsteinsche Behandlung des Themas basierte auf physikalischen Prinzipien, und die mathematischen Grundlagen fehlten. Minkowski, und von ihm unabhängig, Poincaré, formulierten diese Grundlagen, die sich als von entscheidender Bedeutung für die Entwicklung der modernen Physik erwiesen. Die Hauptidee war die Einführung der 4-dimensionalen Raum-Zeit-Koordinaten. Minkowskis berühmter Vortrag auf der Naturforscherversammlung in Köln, 1908, beginnt mit den Worten:

\section{Raum and Zeitt ${ }^{1}$.}

\section{Von Hermaxn Miskowska in Göttingen.}

M. H.! Die Anschaunngen aber Raum und Zeit, die ieh Thnen entwickeln möchte, sind anf experimentell-physikalischem Boden erwschsen. Darin liegt ihre Stirike. Thre Tendenz ist eine radikale. Von Stund' an sollen Raum für sich und Zeit fär sich völlig zu Schatten herabsinken und nur noch eine Art Union der beiden soll Selbetāndig keit bewahren.

In diesem Vortrag führt Minkowksi die Grundbegriffe der Lorentz-Geometrie ein und beschreibt den „MinkowskiRaum“, als Pendant des euklidischen Raumes. Nach Pythagoras, werden im gewöhnlichen euklidischen Raum die Abstände mit der Metrik

$$
d s=\sqrt{d x_{1}^{2}+d x_{2}^{2}+\ldots+d x_{3}^{2}}
$$

gemessen und der Abstand ist das Integral $\int d s$. In der Be- schreibung von Minkowski ist die quadratische Form durch

$$
d \tau=\sqrt{c^{2} d t^{2}-d x_{1}^{2}-\ldots-d x_{3}}
$$

gegeben, wobei $t$ als Zeitkoordinate verstanden wird und $c$ die Fortpflanzungsgeschwindigkeit des Lichtes im leeren Raume ist. Und das ist genau die quadratische Form, die Minkowski als 18-Jähriger untersuchte, als er ganze und rationale Lösungen der Gleichung

$$
c=x_{1}^{2}+x_{2}^{2}+x_{3}^{2}, \quad c \in \mathbb{N},
$$

verstehen wollte!

Die Bewegung eines Teilchens im Minkowski-Raum wird durch eine Kurve beschrieben, deren Länge durch das Integral $\int d \tau$ gegeben wird. Damit diese Länge nicht negativ ist, muss das Teilchen langsamer als die Lichtgeschwindigkeit sein, also muss die Kurve im Inneren des „Lichtkegels“ liegen. Der Ansatz von Minkowski erklärte die äusserst phantastische Hypothese der Längenverkürzung in Richtung der Bewegung und andere Phänomene der Einsteinschen Relativitätstheorie. Den Einwänden von Physikern vorbeugend (Einstein: überflüssige Gelehrsamkeit), schrieb Minkowski:

Über den Begriff des Raumes in entsprechender Weise hinwegzuschreiten, ist auch wohl nur als Verwegenheit mathematischer Kultur einzutaxieren.

Als eine weitere Provokation an die Physiker könnte man auch die Einführung des Postulats der absoluten Welt (oder kurz Weltpostulats) einstufen.

Eine etwas überraschende Anwendung des MinkowskiRaumes ist die Analyse des Flugzeug-Boarding-Problems $[1,2]$. Passagiere werden als Punkte in der Ebene mit Koordinaten $q, r$ dargestellt, wobei $q$ die Position des Passagiers in der Schlange repräsentiert und $r$ die Sitzreihe. Wir können annehmen, dass $q$ und $r$ so normiert sind, dass ihre Werte im Einheitsquadrat liegen. Ein wichtiger Parameter ist die Staukonstante $k$, definiert als Quotient der Anzahl der Passagiere pro Reihe dividiert durch den Abstand zwischen aufeinander folgenden Reihen. Im einfachsten Fall, wenn es keine von der Fluggesellschaft vorgeschriebenen Einstiegsrichtlinien gibt, also wenn $q$ und $r$ voneinander unabhängig sind, können wir die ,eigentliche Zeit" als

$$
d \tilde{\tau}=\sqrt{d q d r+k(1-r) d q^{2}}
$$

definieren. In diesem Model ist die Einstiegszeit, als Funktion der Staukonstante $k$, proportional zur maximalen Länge einer Kurve im Einheitsquadrat bezüglich $d \tilde{\tau}$. Es stellt sich heraus, dass es eine Koordinatentransformation gibt, die von $k$ abhängt, und die $d \tilde{\tau}$ in das $d \tau$ von Minkowski, und die Einstiegszeit in die maximale Eigenzeit überführt. Diese Größen können unter variierenden Boardingszenarien berechnet werden; und diese Berechnungen reduzieren sich in vielen Fällen auf die Analyse verschiedener Aspekte des Minkowski-Raumes.

Und hier ist eine andere Anwendung: In [4] wird ein Algorithmus vorgestellt, der es erlaubt, schneller auf FlashSpeicher zuzugreifen. Die Methode basiert im Wesentlichen auf der Geometrie konzentrischer „Kreise“ im MinkowskiRaum. 
Beim Lesen der Arbeiten und Briefe von Minkowski fällt auf, wie leicht, spielerisch, und fast verträumt er mit Mathematik umgeht. Seine Ideen sind anschaulich und „wirklichkeitsnah“, aber gleichzeitig auch sehr weittragend, was ihm durchaus bewusst war. Seine Festrede auf Dirichlet [8] vermittelt den Eindruck, dass er über sich selbst spricht:

Unablässig war sein Sinnen darauf gerichtet, zwischen getrennt bestehenden Gedankensphären die Brücke zu schlagen und unabhängig gezeitigte Erfolge $\mathrm{zu}$ fruchtbarer Wechselwirkung zu verschmelzen.

Es ist erstaunlich, wie zutreffend seine halb im Scherz hingeworfenen Prophezeihungen erscheinen:

Man hört von der Arithmetisierung ALLER mathematischen Wissenszweige sprechen. Manche halten deshalb die Arithmetik nur noch für eine zweckmässige Staatsverfassung, die sich das ausgedehnte Reich der Mathematik gibt. Ja, zuletzt werden einige in ihr nur noch die hohe Polizei sehen, welche befugt ist, auf alle verbotenen Vorgänge im weitverzweigten Gemeinwesen der Größen und Funktionen zu achten.

Auch heute hört man oft - nicht nur im Reich der Mathematik - vom allgegenwärtigen Einsatz einer besonderen Art der Arithmetik: der Kryptografie.

Ich bin für die Zahlentheorie Optimist und hege still die Hoffnung, daß wir vielleicht gar nicht weit von dem Zeitpunkt entfernt sind, wo die unverfälschte Arithmetik gleichfalls in Physik und Chemie Triumphe feiern wird, und sagen wir z. B., wo die wesentlichen Eigenschaften der Materie als mit der Zerlegung der Primzahlen in zwei Quadrate im Zusammenhang stehend erkannt werden. An jenem Tage werden den Arithmetikern von allen Seiten Huldigungen dargebracht werden.

Auch aus heutiger Sicht ist dieser Optimismus durchaus berechtigt. Erlaubt man die Auffassung, dass der MinkowskiRaum, als Idee, etwas mit Zerlegung von Zahlen in drei Quadrate zu tun hatte, so wäre es durchaus in Ordnung, die (noch ausstehenden) Huldigungen seitens der Fluggesellschaften und der Hersteller von Flash-Speichern an die
Arithmetik weiterzuleiten. Was die Zerlegung von Primzahlen in zwei Quadrate angeht, so hat das etwas mit quadratischer Reziprozität zu tun, und die geometrische Version einer weitreichenden Verallgemeinerung der Reziprozität, das Langlands-Programm, wird in neuester Zeit auch von Physikern studiert.

Vielleicht wird sich, zuletzt, das Land Berlin noch dazu bewegen lassen, die Nichtverlängerung der Anerkennung der Brüder Minkowski zu überdenken.

\section{Literatur}

[1] E. Bachmat, Mathematical adventures in performance analysis: From Storage Systems, Through Airplane Boarding, to Express Line Queues, Series: Modeling and Simulation in Science, Engineering and Technology, Birkhäuser, Basel, 2014.

[2] E. Bachmat, D. Berend, L. Sapir, S. Skiena and N. Stolyarov, Analysis of airplane boarding via space-time geometry and random matrix theory, Journal of physics A: mathematical and general, vol. 39, 453-459, 2006.

[3] M. Bhargava, A positive proportion of plane cubics fail the Hasse principle, arXiv: 1402.1131, 2014.

[4] A. Bhatia, M. Qin, A.R. Iyengar, B.M. Kurkoski, P. Siegel, Lattice based WOM codes for multilevel flash memories, IEEE journal on selected areas in communications, vol. 32(5), 933-945, 2014.

[5] M. Born, My Life: Recollections of a Nobel Laureate, Scribner, New York, 1978.

[6] T. Browning, L. Matthiesen, A. Skorobogatov, Rational points on pencils of conics and quadrics with many degenerate fibres, Annals of Math., vol. 180, 381-402, 2014.

[7] A. Einstein, Zur Elektrodynamik bewegter Körper, Annalen der Physik, vol. 17, 891-921, 1905.

[8] H. Minkowski, Peter Gustav Lejeune Dirichlet und seine Bedeutung für die heutige Mathematik, Jahresbericht der DMV, Band 14, 149-163; in Gesammelte Abhandlungen von Hermann Minkowski, Chelsea Publishing, New York, 1967.

[9] L. Rüdenberg, H. Zassenhaus, Hrsg., Briefe von Minkowski an Hilbert, Springer-Verlag, 1973.

[10] J.-P. Serre, Smith, Minkowski et l'Académie des Sciences, Gaz. Math. No. 56, 3-9, 1993.

[11] J. W. L. Glaisher, ed., The Collected Mathematical Papers of Henry John Stephen Smith, I, II, New York: AMS Chelsea Publishing, 1965.

[12] U. Stammbach, Die Berufung von Hermann Minkowski an das Eidgenössische Polytechnikum in Zürich.

Zuerst in Mitteilungen der Deutschen Mathematiker-Vereinigung 22 (2014), 228-233. Wiederabdruck anlässlich der Diskussion eines dem Andenken von Hermann Minkowski gewidmeten Preises für herausragende wissenschaftliche Leistungen (vgl. S. 57 in diesem Heft).

Prof. Dr. Yuri Tschinkel, Courant Institute of Mathematical Sciences,

New York University, 251 Mercer St., New York, NY 10012, USA

tschinkel@cims.nyu.edu

Yuri Tschinkel, Jahrgang 1964, studierte an der Moskauer Staatlichen Universität und promovierte am Massachusetts Institute of Technology. Danach war er Junior Fellow der Harvard Society of Fellows, Leibniz Fellow der EU an der École Normale Supérieure in Paris, Assistant und Associate Professor an der University of Illinois in Chicago, Visiting Associate Professor an der Princeton University und Gauss-Lehrstuhlinhaber in Göttingen. Er ist jetzt Professor am Courant Institute der New York University und Direktor der Abteilung Mathematik, Physik und Informatik der Simons Stiftung.

Er publizierte etwa 100 Arbeiten auf dem Gebiet der arithmetischen und algebraischen Geometrie. Er ist Herausgeber von 16 Büchern und mehreren Zeitschriften. Er ist Fellow der American Mathematical Society und der American Association for the Advancement of Science. 